$\begin{array}{llr}\text { Universitas } & \text { Vol. } 37 \\ \text { Núm. } 78\end{array}$

\title{
Expresiones clínicas de los trastornos potencialmente malignos en la cavidad oral. Revisión integrativa de la literatura*
}

\author{
Clinical Expressions of Potentially Malignant Disorders in Oral Cavity. Integrative Review of Literature
}

\author{
Eilien Gisek Tovio Martinez ${ }^{\mathrm{a}}$ \\ Universidad de Cartagena, Colombia \\ etoviom@unicartagena.edu.co \\ ORCID: http://orcid.org/0000-0003-3702-2791 \\ Martha Cecilia Carmona Lorduy \\ Universidad de Cartagena, Colombia \\ ORCID: http://orcid.org/0000-0003-3066-2219 \\ Antonio José Díaz-Caballero \\ Universidad de Cartagena, Colombia \\ ORCID: http://orcid.org/0000-0001-9693-2969 \\ Jonathan Harris Ricardo \\ Corporación Universitaria Rafael Núñez, Colombia \\ Universidad de Cartagena, Colombia \\ ORCID: http://orcid.org/0000-0002-4662-0729
}

Héctor E. Lanfranchi Tizeira

Universidad de Buenos Aires, Argentina

ORCID: http://orcid.org/0000-0002-4596-2671
DOI: https://doi.org/10.11144/Javeriana.uo37-78.ecdp Redalyc: http://www.redalyc.org/articulo.oa? id $=231260072005$

Fecha de recepción: 27 Julio 2017 Fecha de aprobación: 04 Junio 2018 Fecha de publicación: 18 Junio 2018

\section{Resumen:}

Antecedentes: Los trastornos potencialmente malignos (TPM) son aquellas situaciones clínicas en la cavidad bucal que presentan peligro de un aumento de malignización neoplásica, debido a la exposición a factores de riesgo o alteraciones genéticas. Se necesita revisar los hallazgos sobre este tipo de trastornos para desarrollar o actualizar guías de práctica clínica idóneas. Objetivo: Identificar, mediante una revisión integrativa de la literatura, la evidencia reciente sobre los TPM de la cavidad bucal y su transformación maligna, a fin de proporcionar recomendaciones de manejo diagnóstico y terapéutico. Métodos: Se buscó literatura sobre el tema en las bases de datos PubMed, Elsevier, SciELO y EMBASE, utilizando la combinación de seis descriptores. Resultados: La búsqueda inicial arrojó 1743 títulos y la muestra consistió en 67 artículos después de aplicar los criterios de inclusión y exclusión. Los TPM identificados fueron liquen plano oral, palatitis nicotínica, hábito de fumar invertido, queilitis actínica, eritroplasia y leucoplasia oral y úlcera traumática crónica. Conclusión: Cada tipo de lesión tiene distinto potencial de malignización, entre los cuales la eritroplasia, el liquen plano oral variante erosivo y la queilitis actínica poseen el mayor riesgo.

Palabras clave: cancerología, eritroplasia, estomatología, lesiones precancerosas, leucoplasia oral, liquen plano oral, malignización, odontología, medicina oral, patología oral, transformación celular neoplásica.

\begin{abstract}
:
Background: Potentially malignant disorders (PMD) are clinical oral cavity conditions that pose an increased risk of neoplastic malignization due to exposure to risk factors or genetic alterations. It is necessary to conduct evidence-based reviews of this type of disorders to develop or update adequate clinical practice guidelines. Purpose: To identify, through an integrative review of literature, recent evidence on PMDs in the oral cavity and their malignant transformation, in order to provide diagnostic and treatment recommendations. Methods: A literature search was carried out in the PubMed, Elsevier, SciELO, and EMBASE, using a combination of six descriptors. Results: The initial search showed 1743 titles and the sample, after applying the inclusion and exclusion criteria, consisted of 67 articles. The PMDs identified were oral lichen planus, nicotinic palatitis, inverted smoking
\end{abstract}

Notas de autor:

a Autora de correspondencia. Correo electrónico: etoviom@unicartagena.edu.co 
habit, actinic cheilitis, oral erythroplakia and leukoplakia, and chronic traumatic ulcer. Conclusion: Each type of lesion has a distinctive malignization potential, of which erythroplakia, oral lichen planus erosive variant, and actinic cheilitis pose the highest risk.

Keywords: dentistry, erythroplakia, malignization, neoplastic cell transformation, oral leukoplakia, oral lichen planus, oral medicine, oncology, oral pathology, precancerous lesions, stomatology.

\section{Introducción}

En 1978, la Organización Mundial de la Salud (OMS) clasificaba los tumores de la cavidad oral en lesiones precancerosas y estados precancerosos, por ser factores de riesgo para la progresión del cáncer. Pindborg y colaboradores en 1997, con la colaboración de la OMS, plantearon una nueva clasificación en la que diversificaron las lesiones precancerosas, tanto por su aspecto clínico como histológico, y detallaron las condiciones precancerosas. Incluyeron la leucoplasia, la eritroplasia, la queilitis actínica y el liquen plano oral (1). Más adelante, en el 2005, la OMS, junto con un comité de expertos del Reino Unido, sugirió remplazar esa terminología por trastornos potencialmente malignos (TPM). Los definieron como aquellos tejidos alterados morfológicamente en los que es más probable que el cáncer ocurra (2). Este término se emplea para describir las alteraciones morfológicas de la mucosa oral que anteceden a una lesión maligna, esto es, condiciones patológicas que implican a la vez un potencial de malignización y un indicador de riesgo de neoplasias malignas (3).

El cuerpo humano actúa de manera continua para mantener el equilibrio; sin embargo, el estrés, la ansiedad, la exposición continua a radiaciones, algunos agentes químicos y el consumo de tabaco, drogas y alcohol generan un desbalance fisiológico y desencadenan alteraciones sistémicas. Con esto se incrementa el riesgo de que se produzca cáncer en cualquier sitio de la cavidad oral $(4,5,6,7)$. Los TPM se identifican por ser lesiones tumorales que, por lo habitual, invaden rápidamente, infiltran, destruyen los tejidos adyacentes y poseen la capacidad de diseminarse a otros sitios en el cuerpo (8). El conocimiento sobre este tipo de lesiones avanza continuamente, por lo que es necesario llevar a cabo revisiones y analizar la evidencia para desarrollar o actualizar guías idóneas de práctica clínica. Por tal razón, el propósito de la presente revisión integrativa de la literatura fue identificar la evidencia reciente sobre TPM de la cavidad bucal y su transformación maligna, y generar recomendaciones de manejo diagnóstico y terapéutico.

\section{Materiales y métodos}

Se hizo una revisión integrativa de la literatura en las bases de datos PubMed, Elsevier, SciELO y EMBASE. La búsqueda incluyó una combinación de descriptores en inglés: potentially malignant disorders AND oral cancer; malignant oral lesions; malignization of oral lesions; oral risk lesions for cancer; y premalignant lesions of the oral cavity. En español/castellano, los términos de búsqueda fueron: trastornos potencialmente malignos $\mathrm{Y}$ cáncer oral; lesiones orales malignas; malignización de lesiones orales; lesiones de riesgo oralpara cáncer; y lesiones premalignas de la cavidad oral. Se establecieron límites de idioma (inglés y español), tipo de artículo (estudios clínicos, estudios de casos y controles, ensayos clínicos, revisiones sistemáticas, metanálisis, casos clínicos y estudios descriptivos), solo estudios en humanos y sin distinción de sexo y edad. Las fechas de publicación indagadas fueron: 1 de enero del 2000 a 31 de diciembre del 2017.

\section{PIOS}

- P-Población: pacientes con liquen plano oral, úlcera traumática crónica, palatitis nicotínica, hábito de fumar invertido, queilitis actínica, eritroplasia y leucoplasia oral.

- I-Intervenciones: diagnóstico clínico de los TPM en la cavidad oral y su transformación maligna.

- O-Objetivo: características clínicas de los TPM en la cavidad oral. 
- S-Estudios incluidos: estudios clínicos, de casos y controles, revisión sistemática, metanálisis, casos clínicos y estudios descriptivos.

Criterios de inclusión

- Artículos en inglés y español en texto completo.

- Estudios publicados entre el 1 de enero del 2000 y el 31 de diciembre del 2017.

- Estudios que brindaran información sobre TPM en la cavidad oral.

- Estudios que brindaran información de lesiones malignas en cavidad oral, transformaciones de lesiones orales en cáncer y cáncer oral.

Criterios de exclusión

- Estudios que proporcionaron información inadecuada.

- Artículos de estudios in vitro.

- Estudios en animales.

- Artículos en un idioma diferente al inglés y español.

- Estudios que incluían infecciones orales.

\section{Resultados}

Los 1743 títulos obtenidos en la búsqueda inicial en las cuatro bases de datos se introdujeron en una hoja de cálculo electrónica. De estos, se descartaron 212, por duplicación; 436, por disponibilidad únicamente del resumen; 319, por título; 561, por no estar relacionados con el tema, y 148, por no cumplir con los criterios de selección. Al final, la muestra consistió en 67 artículos (figura 1). Con ellos se efectuó la revisión integrativa, cuyos resultados se encuentran a continuación, lesión por lesión. 


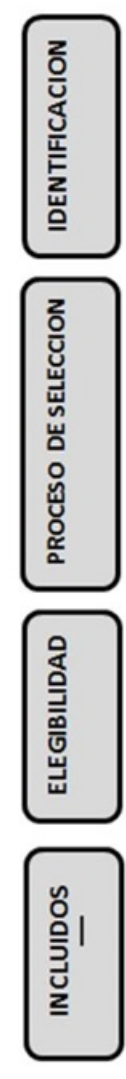

Se identificaron $(n=1.743)$ artículos inicialmente en las bases de datos: PubMed, Elsevier, SciELO y EMBASE

Descartados ( $n=212$ ) por duplicación, $(n=436)$ por estar disponible solo el resumen, $(n=319)$ por título, no relación con el tema $(n=561)$

Se eligieron $(n=215)$ artículos en texto completo

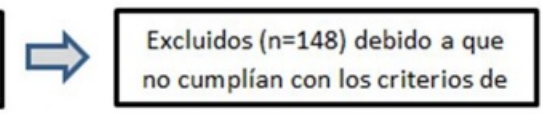

Se incluyeron ( $n=67)$ artículos para el análisis de datos final.

FIGURA 1

Diagrama de flujo

Fuente: elaboración propia.

\section{Liquen plano oral}

El liquen plano oral (LPO) es una enfermedad mucocutánea crónica, de carácter inflamatorio, recurrente $(9,10,11,12)$, que presenta una incidencia del $2 \%$ en la población general. Es más frecuente en mujeres $(60-70$ $\%)$, con un pico de prevalencia entre los 50 y los 55 años $(9,10,11,12,13)$. Aunque aparece más en la mediana edad, también puede desarrollarse en niños y jóvenes (14).

La etiología del LPO es desconocida. No obstante, se atribuye a factores inmunológicos, sin descartar factores agravantes como genéticos, infecciosos, neurológicos y psicológicos (10,15). Su fisiopatología es de tipo autoinmune mediada por linfocitos $\mathrm{T}(9,10,11,16)$, que afectan el epitelio escamoso estratificado $(17,18)$. Ocasiona un ataque inmunológico a los queratinocitos basales originado por factores exógenos o endógenos, que provocan una transformación en la respuesta a autoantígenos (14).

El LPO presenta varias manifestaciones clínicas que se clasifican como típico (reticular) y atípico (forma atrófica, en placa, erosiva, bullosa y mixta) (19). Durante la exploración y para su diferenciación clínica, hay que tener en cuenta el tipo de lesión, la localización, su bilateralidad y simetría (20,21). La variante más frecuente es la reticular, que se observa como manchas blancas, lineales en forma estrellada, de red o arboriformes (estrías de Wickham), que no se desprenden al ser raspadas. Se localiza especialmente en mucosa yugal de manera bilateral, simétrica y es asintomático $(17,22,23)$.

La variante atípica atrófica se caracteriza por presentar zonas rojas atróficas en las que hay una solución de continuidad del epitelio. Se localiza frecuentemente en la lengua, la mucosa yugal, la encía, el fondo de surco de la mucosa labial y el piso de la boca. Cuando se presenta en la mucosa yugal, las lesiones pueden ser 
bilaterales (figura 2). En la forma atípica erosiva se presentan erosiones de cloro rojo intenso a veces sangrantes, localizadas en cualquier sitio de la mucosa oral. También se aprecian ulceraciones. La sintomatología es característica. Esta forma clínica es la que con mayor frecuencia puede sufrir transformación maligna hacia un carcinoma escamocelular $(17,24,25)$.

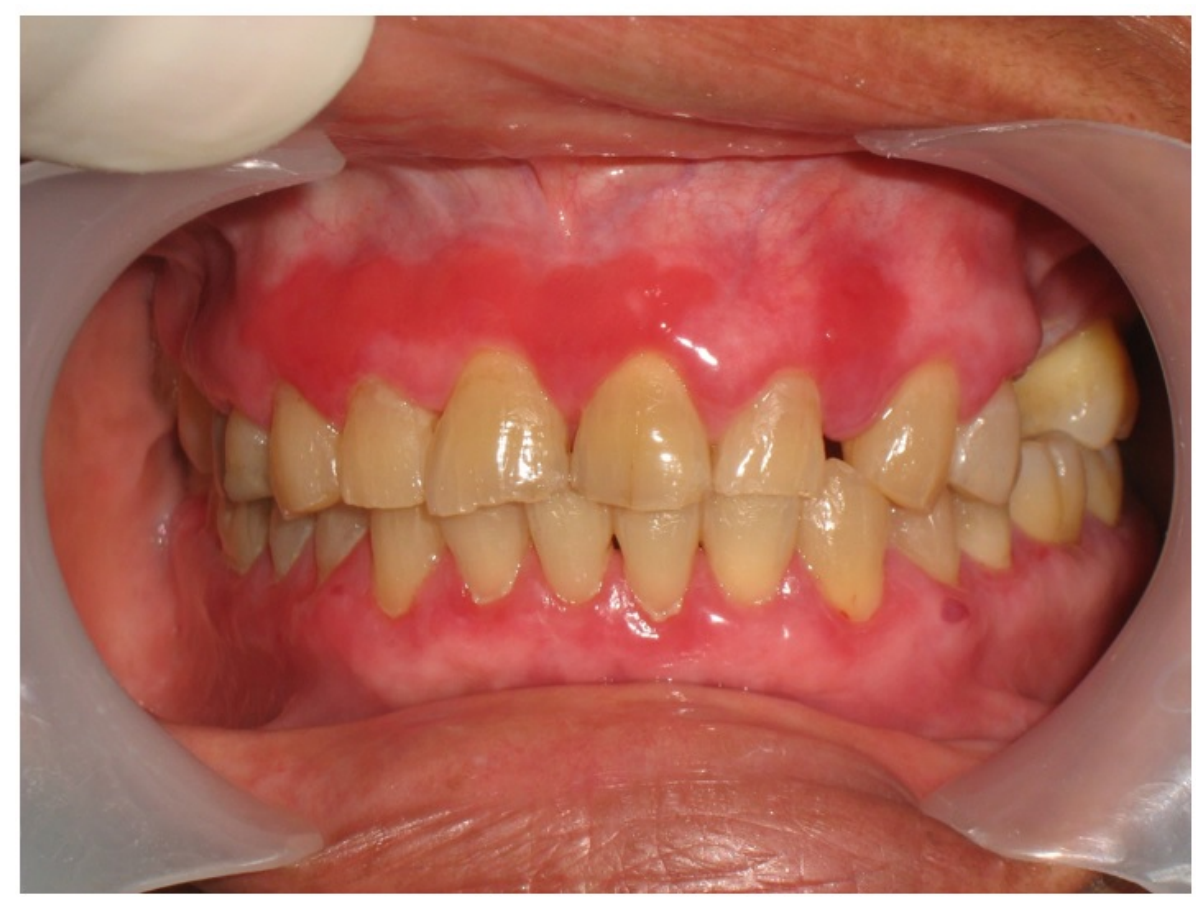

FIGURA 2

Liquen atípico atrófico en el maxilar superior, color rojo localizado en la encía marginal y adherida Fuente: elaboración propia.

Entre las principales características histológicas del LPO se encuentran: hiperqueratosis y acantosis, degeneración hidrópica de la capa basal, infiltrado inflamatorio en banda en la dermis que abraza la lámina basal y es paralelo al epitelio $(10,14,17)$. La presencia y gravedad de la displasia epitelial son primordiales para valorar el riesgo de malignización de esta lesión en la mucosa oral $(9,10,13)$.

El LPO es un TPM susceptible de transformarse en una neoplasia si no se trata inicialmente, lo cual podría tener relación con los sucesos contradictorios que ocurren en la regulación del ciclo de las células epiteliales lesionadas (13). Tomaz y colaboradores (26), en un estudio con 85 pacientes, se refirieron al caso de un paciente que presentó un carcinoma de células escamosas. Por otra parte, durante el diagnóstico histopatológico, tres pacientes con LPO erosivo mostraron atipia celular moderada, lo que confirmó el potencial de transformación maligna del LPO. En una revisión sistemática realizada por Cok y colaboradores (27) se informó de una incidencia de malignización en menos del $1 \%$ y se destacó el potencial de transformación maligna del LPO atrófico erosivo. Por otra parte, Almirón y colaboradores (28) observaron que el LPO puede malignizarse en un $5 \%$, lo cual depende del tipo y del grado de displasia epitelial que presente. En el 2004, Aguas y Lanfranchi (19) reportaron 491 casos de LPO atípico, entre los que se encontró una transformación maligna de alta significancia equivalente al 6,3\%.

\section{Leucoplasia oral}

La OMS define la leucoplasia oral como como una lesión de predominio blanco que no se puede caracterizar clínica ni patológicamente como ninguna otra enfermedad sin tener en cuenta los criterios histológicos (29). Su incidencia oscila entre el 0,2 \% y el 17 \% de la población. La distribución por sexo varía de acuerdo con 
factores de riesgo como tabaquismo y consumo de alcohol (30,31). Algunos estudios dicen que se presenta en una proporción 3:1 entre hombres y mujeres y es más predominante hacia la cuarta o hacia la sexta década de la vida (32,33).

Existen factores externos que predisponen a la patología. Por ello es importante considerar aquellos hábitos como el consumo de alcohol, la masticación de tabaco y el uso de algunos productos químicos. Es importante no dejar de lado la asociación ente microrganismos de tipo fúngico como Candida albicans y virus del tipo del papiloma humano, los cuales favorecen la aparición de TPM $(29,33,34)$.

Clínicamente, la leucoplasia se observa como una lesión única en forma de mancha placa o verrugosidad localizada, que abarca un amplia área de la mucosa oral. Se clasifica en homogénea y no homogénea. La leucoplasia homogénea muestra un color blanco uniforme, patrón que por lo general no está acompañado de infección por Candida, y suelen ser asintomáticas (35). Entre tanto, la no homogénea es una lesión predominantemente blanca, de superficie irregular, sintomática y existen tres tipos: eritroleucoplasia, nodular y exofítico. La variante eritroleucoplasia es una lesión blanca en la que se presentan zonas rojas; en la leucoplasia nodular se presentan nódulos ligeramente elevados y redondos; en la leucoplasia exofítica la lesión es verrugosa blanca con proyecciones irregulares filiformes (figura 3) $(31,32,33,34,36,37)$.

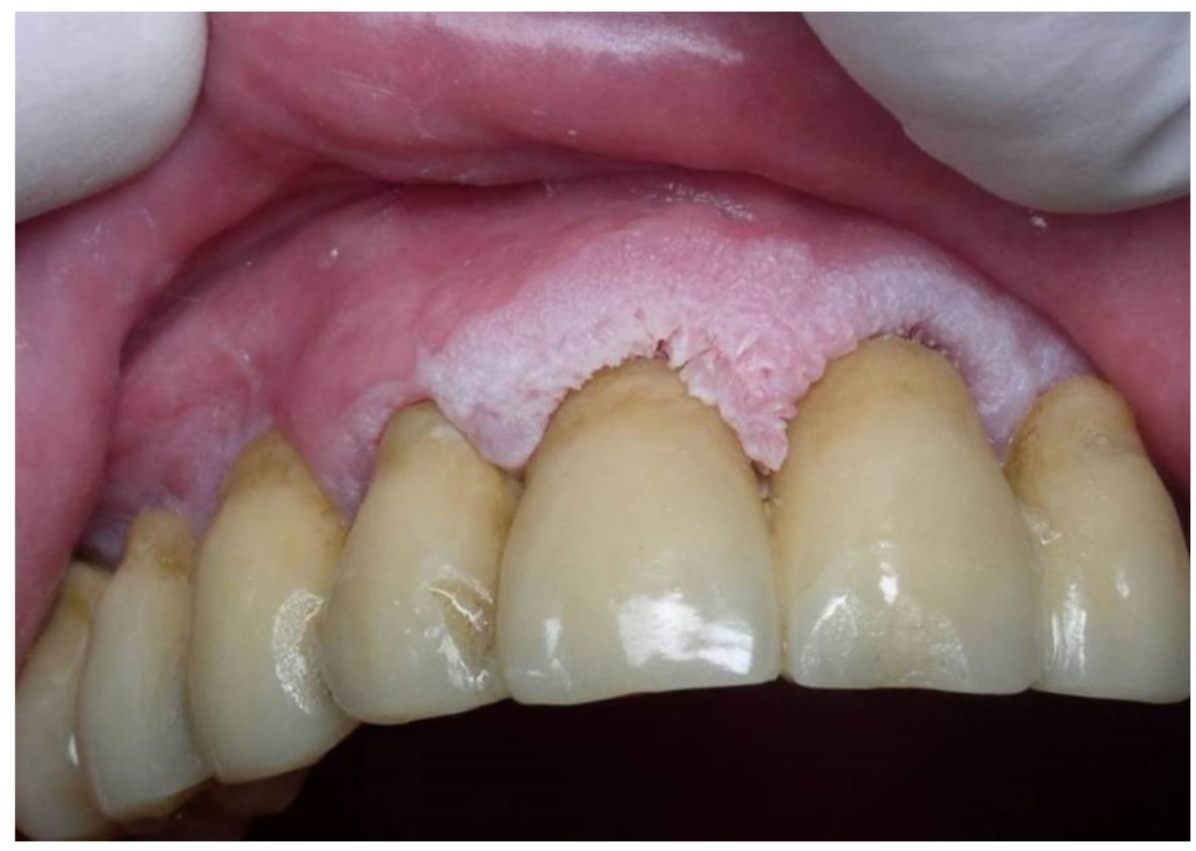

FIGURA 3

Leucoplasia verrugosa en encía marginal y adherida, zona anterosuperor Fuente: elaboración propia.

Los análisis citológicos e histopatológicos de la leucoplasia oral muestran desde una hiperqueratosis sin displasia epitelial hasta una displasia severa con fenómenos de atrofia o hiperplasia epitelial (31). Se puede observar la expresión de un trastorno de maduración en el epitelio oral. Además, presentan características morfológicas y citológicas semejantes a las del carcinoma de células escamosas, con la diferencia de que no hay infiltración del tejido conectivo (38).

Martorell-Calatayud y colaboradores, en el 2009 (31), informaron que el porcentaje de malignización de la leucoplasia fluctúa entre el $1 \%$ y el $18 \%$. Petti (39), por su parte, en una revisión sistemática de literatura sobre la leucoplasia oral, estimó un índice de transformación maligna anual del 1,36\%. Lee y colaboradores (40), en un estudio en población taiwanesa, hallaron un riesgo 2,72 veces mayor de las leucoplasias ubicadas en la lengua hacia carcinomas que en aquellas ubicadas en otros sitios de la cavidad bucal. La prevalencia fue 4-7 veces mayor en las leucoplasias orales no homogéneas (29). Van der Waal (41) destacó una serie de factores de 
riesgo que potencializan la transformación maligna de la leucoplasia. Entre ellos menciona el sexo femenino, el tiempo de evolución, la localización en lengua y piso de boca, las lesiones mayores de $4 \mathrm{~cm}$, las leucoplasias de tipo no homogéneos y la presencia de displasia epitelial.

\section{Eritroplasia oral}

De acuerdo con la OMS, la eritroplasia oral es una placa de color rojo intenso que no se puede caracterizar clínica ni patológicamente como ninguna otra enfermedad definible (42). La etiopatogenia aún es inespecífica. Sin embargo, se asocia al consumo de tabaco y alcohol, así como a la exposición a agentes carcinógenos, infecciones virales, dieta baja en antioxidantes y factores genéticos, que se describen como factores coadyuvantes para su transformación (43). Epidemiológicamente, estas lesiones se presentan entre el $0,2 \%$ y el $0,83 \%$ de la población, con predominio por el sexo masculino, entre la cuarta y sexta décadas de la vida (44).

Se caracteriza por ser una lesión eritematosa como una mancha, placa o, en algunos casos, atrofia, que puede ser plana o deprimida, de superficie lisa o con una leve elevación, de bordes bien definidos, usualmente única, no mayor de $1,5 \mathrm{~cm}, \mathrm{y}$ de aspecto inflamatorio. Se ubica sobre una mucosa habitualmente atrófica, sin presencia de queratina, con una tonalidad rojiza a causa de la fina capa de epitelio que trasluce la microvascularización. Puede o no haber presencia de un componente leucoplásico que, cuando está presente, se denomina eritroleucoplasia (figura 4). Esta lesión, por lo general, es asintomática; sin embargo, algunas veces suele acompañarse con sensación de ardor o dolor (45). Los sitios más afectados son el piso de la boca, el paladar blando, la mucosa yugal, la cara ventral de lengua y las amígdalas (43).

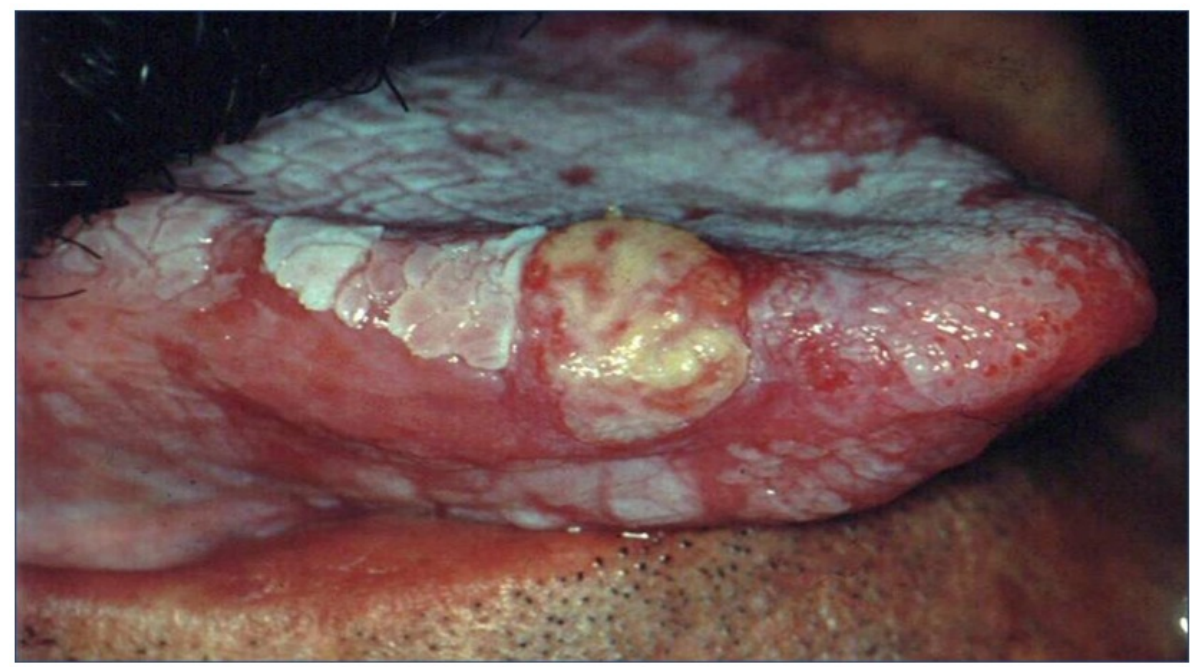

FIGURA 4

Eritroleucoplasia con zonas rojas y blancas en la cara dorsal, ventral de lengua En el borde lateral derecho presenta una lesión tumoral con diagnóstico de carcinoma escamocelular Fuente: elaboración propia.

La forma clínica de la eritroplasia varía según el grado de inflamación que presente y su aspecto granular. Se manifiesta como una lesión de color rojo-aterciopelado de mucosa normal o en algunos casos queratósica, de superficie granular, que sangra con facilidad. Es inusual encontrar en estas lesiones áreas ulceradas, duras y de crecimiento exofítico (46).

En cuanto a la histología de la eritroplasia oral, se puede observar un epitelio delgado atrófico con cambios displásicos e infiltrado inflamatorio. Muestran cambios que van desde una displasia moderada hasta un carcinoma invasivo de células escamosas $(47,48)$. 
La eritroplasia oral presenta una tasa de malignización mayor que los demás trastornos. En un reporte presentado por Reichart y Phillipsen, en el 2005 (49), se afirma que el porcentaje de transformación de la eritroplasia oral oscila entre un $14 \%$ y un $50 \%$. El alto riesgo de transformación maligna se fundamenta en la presentación histológica de la eritroplasia oral de carcinomas in situ, epitelio con displasia severa o un carcinoma microinvasivo, que es la manifestación más temprana. Su transformación es rápida, por lo que debe tratarse a tiempo (45). Un estudio publicado por Noonan y Kabani, en el 2005 (50), se indica que aproximadamente el $16 \%$ de las lesiones de carcinoma oral in situ se diagnosticaron como eritroplásicas y alrededor del $9 \%$ fue una combinación de eritroleucoplasia.

\section{Queilitis actínica}

La queilitis actínica es un TPM del labio que ocurre como respuesta a una exposición prolongada a la radiación ultravioleta. Se evidencian alteraciones de la mucosa y semimucosa labial con mayor predominio en la superficie del labio inferior (51). Epidemiológicamente, esta lesión se expresa con mayor frecuencia en pacientes del sexo masculino, de tez blanca, que superan la cuarta década de la vida. Entre la población de riesgo se ubican quienes trabajan al aire libre y han estado crónicamente expuestos a la radiación ultravioleta (52). Es importante establecer aquellos factores que causen irritación de forma directa y crónica como el hábito de fumar, traumatismos repetitivos y quemaduras; sin embargo, el principal factor causal es la exposición a la radiación ultravioleta, que ocasiona deterioro del colágeno y desintegración de la molécula de vitamina A. Esto desencadena inmunosupresión local y producción de radicales libres de oxígeno, lo que origina daños en el ácido ADN y desencadena lesiones con potencial de transformación maligna (3).

Clínicamente se evidencian áreas secas, fisuradas y con escamas sobre la semimucosa labial inferior, debido a que el labio está más expuesto a la radiación ultravioleta y su epitelio es más delgado, pues posee una fina capa de queratina con menor cantidad de melanina (53). Algunas veces están acompañados de ampollas características de quemadura en su forma aguda. El labio se observa hipotónico, con descamación de la semimucosa labial y xerosis. Pueden encontrarse áreas atróficas, leucoplásicas, erosiones y costras (figura 5), con periodos de mejoramiento y recidiva. Puede también presentar dolor y sangrado. Un aspecto relevante es la pérdida del borde bermellón, es decir, no hay una clara separación entre la semimucosa labial y la piel (54). 


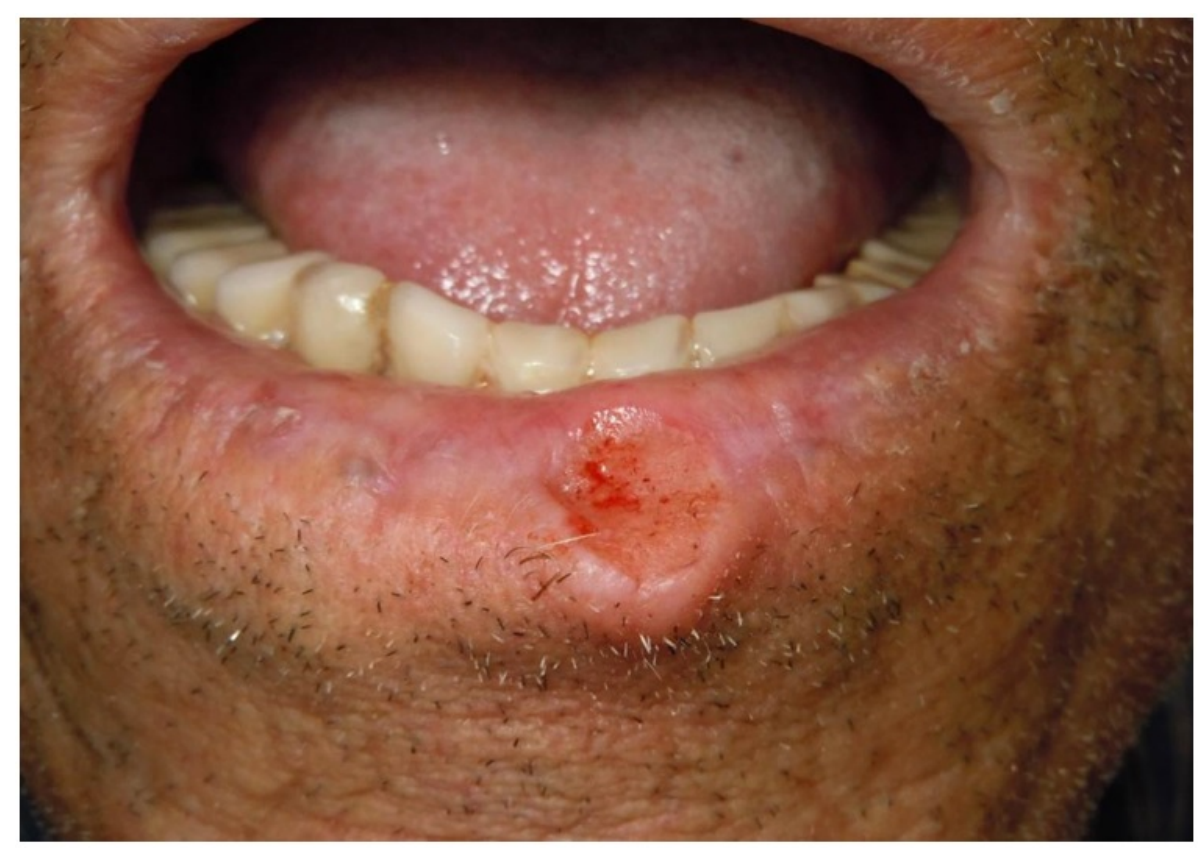

FIGURA 5

Úlcera en semimucosa labial inferior y borde bermellón en paciente con queilitis actínica Fuente: elaboración propia.

La queilitis actínica se puede clasificar en exfoliativa o descamativa y abrasiva o erosiva, de acuerdo con los hallazgos clínicos. La queilitis exfoliativa se caracteriza por la presencia de escamas que sangran con facilidad (54). La queilitis abrasiva presenta una lesión erosiva rojiza bien delimitada, de aproximadamente 5-10 mm de diámetro, con una superficie brillante y lisa. Tiende a recidivar. En algunas ocasiones, hay presencia de costras hemáticas y queilitis fisurada en la que se observan fisuras verticales desde la semimucosa a la mucosa o a la piel (52). Histopatológicamente, se presenta una alteración en la maduración del epitelio con atipia citológica variable, mitosis en los distintos estratos, hiperqueratosis orto y paraqueratósica, infiltrado inflamatorio bajo la mucosa y, en algunos casos, elastosis solar (55).

Es necesario llevar un seguimiento continuo de esta patología y determinar su tiempo aproximado de aparición, así como un examen clínico y la palpación de los labios para identificar un posible endurecimiento de la lesión. La queilitis actínica tiene potencial de malignizarse a carcinoma escamocelular entre el $1 \%$ y el $3 \%$ de los casos en la cavidad bucal y es una de las principales causas de cáncer de labio (53). En un estudio publicado por Sánchez Ferra y colaboradores (54) se indicó que la tasa de transformación maligna de la queilitis actínica se encuentra entre el $0,75 \%$ y el $0,96 \%$ por persona al año. El número total de lesiones en el labio, en un lapso de aproximado de 10 años, constituye de un $13 \%$ al $20 \%$ y señala que el carcinoma espinocelular del labio producto de una queilitis actínica está más predispuesto a originar metástasis (54).

\section{Palatitis nicotínica, hábito de fumar invertido}

El hábito de fumar invertido (HFI), conocido como tabaquismo invertido, consiste en ubicar el extremo encendido del tabaco o cigarrillo dentro de la boca y al cierre labial el fumador aspira el humo. Cuando el tabaco o cigarrillo se encuentra en la cavidad bucal de forma invertida, puede alcanzar temperaturas hasta de $760^{\circ} \mathrm{C}$. El aire intraoral puede tener temperaturas de hasta $120^{\circ} \mathrm{C}(56)$.

La palatitis nicotínica o lesión en paladar de pacientes con HFI es una patología cuyo aspecto clínico es variable. Se ha observado una relación entre el no consumo y la resolución de las lesiones. Esto sucede cuando una persona suspende el hábito de fumar; por ello se atribuye como agente etiológico el tabaco o cigarrillo 
fumado de forma no convencional (57). El HFI es más frecuente en el sexo femenino y en zonas rurales de Latinoamérica e India (58).

Dentro de las manifestaciones clínicas, inicialmente se evidencia una reacción de tipo eritematoso, que luego evoluciona a mancha, placa o verrugosidad de color blanco grisáceo en el paladar duro. Está acompañada de pápulas rojas de $1-5 \mathrm{~mm}$ de diámetro, con engrosamiento umblicado focal alrededor de los orificios de salida de los conductos de las glándulas salivales palatinas menores (figura 6) (19).

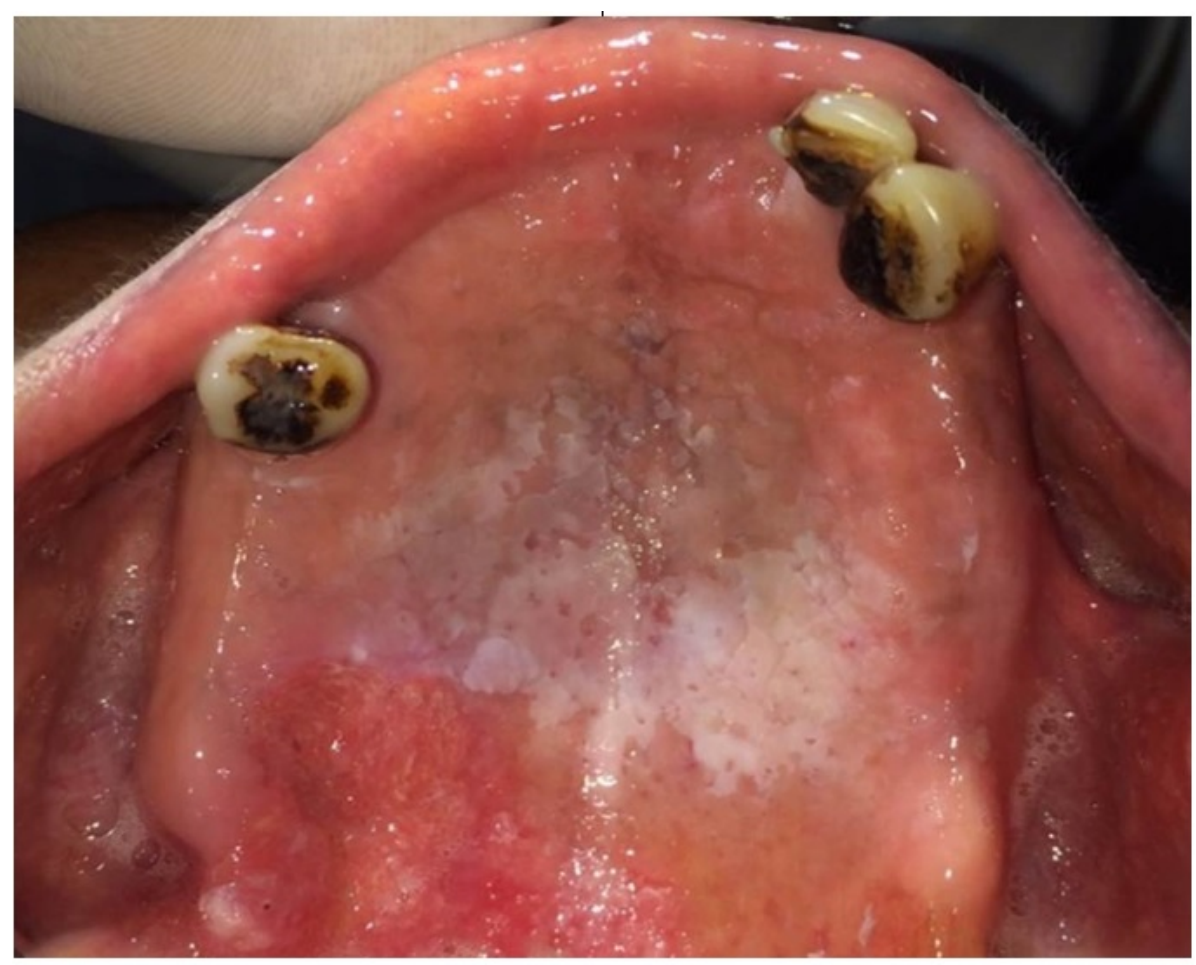

FIGURA 6

Palatitis nicotínica que afecta toda la extensión del paladar duro, con carcinoma escamocelular en paciente con tabaquismo invertido Fuente: elaboración propia.

Histológicamente, se caracteriza por un epitelio acantósico hiperqueratinizado. Los conductos salivales pueden presentar metaplasia escamosa y el tejido conjuntivo subyacente puede aparecer con un infiltrado inflamatorio crónico, discreto, atrofia de acinos y cicatriz (59).

El HFI debe considerarse un indicador potencial de alteraciones epiteliales importantes en otros sitios (59). Es uno de los factores de riesgo más importantes para el progreso del cáncer bucal. Por lo general, se trata de una lesión reversible cuando se suprime el factor causal, pero puede sufrir eventualmente una transformación maligna, por lo que se considera un TPM. En el 2008, Álvarez-Gómez y colaboradores (60) reportaron un estudio que se realizó en el departamento de Sucre, Colombia. El 63 \% de la población era fumadora invertida y se identificaron 122 casos de cáncer oral. Asimismo, mencionaron que en la ciudad de Montería, departamento de Córdoba, se diagnosticaron 207 casos de cáncer oral, el 41,5\% de los cuales se presentó en fumadores invertidos de la zona. Otro estudio realizado por Hashibe y colaboradores (3) analizó el consumo de tabaco en relación con un grupo de lesiones en cavidad bucal. Mostró un mayor porcentaje de DPM (41,2 $\pm 35 \%$ ) en una población de fumadores invertidos. 


\section{Úlcera traumática crónica}

Es importante tener en cuenta las perspectivas de autores como Aguas y Lanfranchi (19), Pérez y colaboradores (61), Piemonte y colaboradores (62) y Balkwill y Mantovani (63). Ellos consideran que la úlcera traumática debe incluirse entre los TPM, pues tanto sus características clínicas como su etiología son factores que pueden incurrir en alteraciones de los tejidos afectados.

La úlcera traumática crónica (UTC) es una pérdida de sustancia profunda y crónica de la mucosa que se presenta con un área central amarillenta o sangrante rodeada de un halo eritematoso y bordes crateriformes, que no tiende a cicatrizar. Se origina de afuera hacia adentro y tiene tamaño y profundidad variables. La UTC es una lesión muy frecuente en la cavidad oral (64). Su etiología es el trauma mecánico crónico y persistente ocasionado por dientes con bordes cortantes, prótesis en mal estado o alimentos muy duros (65). Estas lesiones pueden cicatrizar si se retira el agente causal de inmediato. Sin embargo, algunas llegan al estado crónico. Epidemiológicamente, se puede encontrar en ambos sexos, con variabilidad respecto de la edad. Usualmente, las UTC se ubican hacia los bordes laterales de la lengua, la cara interna de las mejillas, los labios y esporádicamente el paladar, esto es, zonas de alto roce dentario (65) (figura 7).

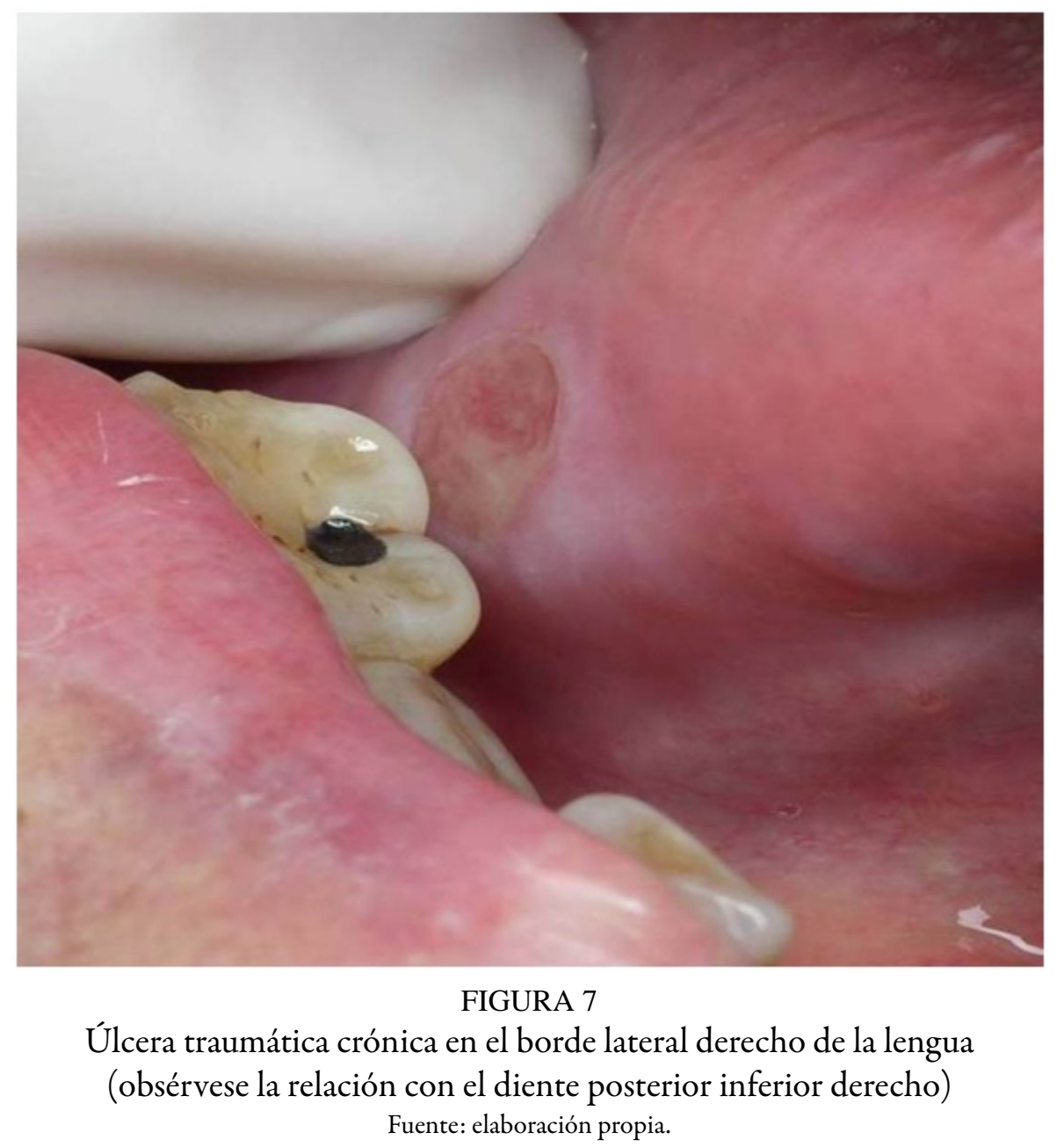

Desde el punto de vista histopatológico, las UTC muestran una solución de continuidad del epitelio y corion con tejido hiperplásico en los márgenes y un tejido de granulación mixto a lo largo de la base y en profundidad. También se evidencia un exudado fibrinoso que cubre el conectivo expuesto, con un infiltrado inflamatorio con polimorfonucleares, linfocitos y células plasmáticas (66). 
Se debe tener en cuenta que el carcinoma de lengua tiene una prevalencia del $20 \%$ entre los carcinomas orales. En el estudio realizado por Aguas y Lanfranchi (19) de 107 casos de UTC, el 6,42 \% evolucionó a carcinoma, primordialmente aquellos que se hallaron en el borde de la lengua. Valente y colaboradores, en el 2016 (67), reportaron el caso de una mujer de 65 años de edad con una lesión ulcerada en la mucosa del reborde alveolar de origen traumático protésico relacionado con una prótesis removible desadaptada. La lesión tenía un periodo de evolución de 6 meses y al análisis histopatológico reveló un carcinoma invasivo ulcerado. Casos como este sugieren la transformación maligna de las UTC.

\section{Discusión}

Los TPM dan lugar a la aparición de carcinomas orales y constituyen el $3 \%$ del cáncer bucal. Aun cuando se ha avanzado en la terapéutica, la tasa de sobrevivencia luego de 5 años sigue siendo del $50 \%$ al $60 \%$. Por esto, la prevención y el abordaje temprano de estas patologías son fundamentales (7).

Luego de una exhaustiva búsqueda de literatura, es notoria la falta de estudios actuales sobre la malignización de los TPM en la cavidad oral y el análisis de sus factores de riesgo. Aún quedan interrogantes por resolver asociados con los mecanismos de malignización, teniendo en cuenta que el desarrollo de estas patologías orales suele tener un factor desencadenante. Existen muchos estudios que abordan el tema. Sin embargo, según toda la información recopilada, esta puede ser una de las primeras revisiones integrativas de la literatura en que se aborden todos los TPM y su transformación maligna. Este es un tema de interés para la disciplina odontológica y la salud pública.

Los resultados de este análisis permiten reconocer y visualizar los aspectos generales de los TPM y sus agentes causales. Del mismo modo, brindan la oportunidad de tener una mente abierta a un mundo de gran desconocimiento en relación con estas patologías orales y permite a odontólogos generales y especialistas tener particular cuidado con pacientes que presenten patologías.

\section{Conclusión}

Entre los 67 artículos revisados se identificaron las siguientes TPM: liquen plano oral, palatitis nicotínica, hábito de fumar invertido, queilitis actínica, eritroplasia y leucoplasia oral y úlcera traumática crónica. Cada tipo de lesión tiene un distinto potencial de malignización, entre los cuales la eritroplasia, el liquen plano oral variante erosivo y la queilitis actínica poseen el mayor riesgo.

\section{Recomendaciones}

Es necesario continuar realizando estudios que evidencien los mecanismos de malignización de los TPM, así como la formación e instrucción de los profesionales de salud en esta área.

\section{Referencias}

1. Warnakulasuriya S, Johnson NW, van der Waal I. Nomenclature and classification of potentially malignant disorders of the oral mucosa. J Oral Pathol Med. 2007 Nov; 36(10): 575-80. https://doi.org/10.1111/j.1600-0714.200 7.00582.x

2. Blanco Carrión A, Otero Rey EM, Peñamaría-Mallón M, Blanco-Carrión A. Desórdenes orales potencialmente malignos. Manifestaciones clínicas. RCOE [internet]. 2013; 18(2): 70-82. Disponible en: https://dialnet.uniri oja.es/servlet/articulo?codigo $=6287919$ 
3. Hashibe M, Brennan P, Benhamou S, Castellsague X, Chen C, Curado MP, Dal Maso L, Daudt AW, Fabianova E, Fernandez L, Wünsch-Filho V, Franceschi S, Hayes RB, Herrero R, Koifman S, La Vecchia C, Lazarus P, Levi F, Mates D, Matos E, Menezes A, Muscat J, Eluf-Neto J, Olshan AF, Rudnai P, Schwartz SM, Smith E, Sturgis EM, Szeszenia Dabrowska N, Talamini R, Wei Q, Winn DM, Zaridze D, Zatonski W, Zhang ZF, Berthiller J, Boffetta P. Alcohol drinking in never users of tobacco, cigarette smoking in never drinkers, and the risk of head and neck cancer: pooled analysis in the International Head and Neck Cancer Epidemiology Consortium. J Natl Cancer Inst. 2007 May 16; 99(10): 777-89. https://doi.org/10.1093/jnci/djk179

4. Laplace BN, Legrá SM, Fernández J, Quiñones D, Piña L, Castellanos L. Enfermedades bucales en el adulto mayor. Correo Cientif Med. 2013; 17(4): 477-88.

5. Gonzáles R, Herrera B, Osorio M, Madrazo OD. Principales lesiones bucales y factores de riesgo presentes en población mayor de 60 años. Rev Cub Estomatol. 2010; 47(1): 105-14.

6. Galbiatti AL, Padovani-Junior JA, Maníglia JV, Rodrigues CD, Pavarino EC, Goloni EM. Head and neck cancer: causes, prevention and treatment. Braz J Otorhinolaryngol. 2013; 79(2): 239-47.

7. Cruz PM, Niño A, Batista K, Miguel-Soca P. Factores de riesgo de cáncer bucal. Rev Cub Estomatol. 2016; 53(3): $128-45$.

8. Syrjänen S, Lodi G, von Bültzingslöwen I, Aliko A, Arduino P, Campisi G, Maeda H. Human papillomaviruses in oral carcinoma and oral potentially malignant disorders: a systematic review. Oral Dis. 2011; 17(1): 58-72.

9. Aguado JM, Rubio D. Apoptosis y liquen plano oral: situación actual. Av Odontoestomatol [internet]. 2009; 25(1): 11-18. Disponible en: http://scielo.isciii.es/pdf/odonto/v25n1/original1.pdf

10. Blanco A, Otero E, Peñamaría M, Diniz M. Diagnóstico del liquen plano oral. Av Odontoestomatol. 2008; 24(1): 11-31.

11. Colonia A, Vélez LF. Liquen plano oral. CES Odontol. 2011; 24(2): 79-86.

12. Urbizo J. Liquen plano bucal y displasia epitelial. Rev Cub Estomatol. 2013; 49(2): 162-70.

13. Cerero R. Malignización del liquen plano oral. Av Odontoestomatol. 2008; 24(1): 97-103.

14. Matesanz P, Bascones A. Liquen plano: revisión de la literatura actual. Av Odontoestomatol. 2009; 25(2): 99-114.

15. Lee KH, Polonowita AD. Oral white lesions: pitfalls of diagnosis. Med J Aust. 2009; 190(5): 274-7.

16. Hernández S, Pratt I, Rodríguez M, Rodríguez M. Oral lichen planus versus acute herpetic gingivostomatitis. Rev Cienc Med. 2013; 17(6): 188-95.

17. Bascones C, Gonzales-Moles MA, Carrillo de Albornoz A, Bascones A. Liquen plano oral (I). Aspectos clínicos, etiopatogénicos y epidemiológicos. Av Odontoestomatol. 2006; 22(1): 11-9.

18. Rodríguez OL. Manifestaciones mucocutáneas del liquen plano: Revisión bibliográfica. Rev Cub Estomatol. 2002; 39(2): 157-86.

19. Aguas SC, Lanfranchi HE. Lesiones premalignas o cancerizables de la cavidad oral. Rev Fac Odontol (UBA). 2004; 19(47): 24-6.

20. Jahanshahi G, Ghalayani P, Maleki L. Mast cells distribution and variations in epithelium thickness and basement membrane in oral lichen planus lesion and oral lichenoid reaction. Dent Res J (Isfahan). 2012; 9: 180-4.

21. Upadhyay RB, Carnelio S, Shenoy RP, Gyawali P, Mukherjee M. Oxidative stress and antioxidant defense in oral lichen planus and oral lichenoid reaction. Scand J Clin Lab Invest. 2010; 70: 225-8.

22. Bermejo-Fenoll A, López-Jornet P. Liquen plano oral. Naturaleza, aspectos clínicos y tratamiento. RCOE. 2004; 9(3): 395-408.

23. Márquez M, Estrada G, González E, Medina L, Jaca A. Manifestaciones bucales del liquen plano. Medisan. 2013; 17(11): 8023-30.

24. Eisen D, Carrozzo M, Bagan Sebastian JV, Thongprasom K. Number V Oral lichen planus: clinical features and management. Oral Dis. 2005 Nov; 11(6): 338-49.

25. Edwards PC, Kelsch R. Oral lichen planus: clinical presentation and management. J Can Dent Assoc. 2002 Sep; 68(8): 494-9. 
26. Tomaz A, Jacomacci WP, Quinto SJ, Veltrini VC, Iwaki VC, Tolentino SE. Potencial de transformación maligna del liquen plano oral: estudio retrospectivo. Int J Odontostomatol. 2015; 9(3): 511-17. http://dx.doi.org/10.4 067/S0718-381X2015000300025

27. Cok S, Cok CC, Bascones-Martínez A. Malignización del liquen plano oral (LPO). Av Odontoestomatol. 2015. 31(5):323-9.

28. Almirón MS, Rosende RO, Zamudio ME, Gili MA. Valoración de la citología exfoliativa como método diagnóstico a propósito de un carcinoma escamoso de lengua. ODN. 2016; 4(11): 61-8.

29. Chandran R, Meer S, Feller L. Oral leukoplakia in a South African sample: a clinicopathological study. Oral Dis. 2013; 19(6): 592-7.

30. Martínez-Sahuquillo Márquez A, Gallardo Castillo I, Cobos Fuentes MJ, Caballero Aguilar J, Bullón Fernández P. La leucoplasia oral. Su implicación como lesión precancerosa. Av Odontoestomatol. 2008; 24(1): 33-44.

31. Martorell-Calatayud A, Botella-Estrada R, Bagán-Sebastián JV, Sanmartín-Jiménez O, Guillén-Barona C. La leucoplasia oral: definición de parámetros clínicos, histopatológicos y moleculares y actitud terapéutica. Actas Dermosifiliogr. 2009; 100(8): 669-84.

32. Escribano-Bermejo M, Bascones-Martínez A. Leucoplasia oral: Conceptos actuales. Av Odontoestomatol. 2009 Mar-Abr; 25(2): 83-97.

33. Medina M, Carmona M, Álvarez P, Díaz-Caballero A. Leucoplasia asociada al hábito de fumar invertido: presentación de un caso clínico. Av Odontoestomatol [internet]. 2015; 31(4): 261-6. Disponible en: http://sc ielo.isciii.es/pdf/odonto/v31n4/original2.pdf

34. Freitas MD, Blanco A, Gándara P, Antúnez J, García A, Gándara JM. Clinicopathologic aspects of oral leukoplakia in smokers and nonsmokers. Oral Surg Oral Med Oral Pathol Oral Radiol Endod. 2006; 102(2): 199-203.

35. Lee JJ, Hong WK, Hittelman WN, Mao L, Lotan R, Shin DM, Benner SE, Xu XC, Lee JS, Papadimitrakopoulou VM, Geyer C, Perez C, Martin JW, El-Naggar AK, Lippman SM. Predicting cancer development in oral leukoplakia: ten years of translational research. Clin Cancer Res. 2000 May; 6(5): 1702-10.

36. Pérez Caffarena M, Cossetti Olivera L, García Corti S. Lesiones cromáticas de la cavidad bucal; lesiones blancas, rojas y pigmentadas. Segunda parte: Lesiones blancas. Actas Odontol. 2016; 3(1): $42-53$.

37. Labori D. Predicción del riesgo de leucoplasia bucal en personas mayores de 60 años. Medisan [internet]. 2012; 16(10): 1548-53. Disponible en: https://www.redalyc.org/articulo.oa?id=368448459010

38. Esparza GC. Valoración de los diferentes tratamientos empleados en la leucoplasia oral. Av Odontoestomatol. 2008; 24(1): 105-9.

39. Petti S. Pooled estimate of world leukoplakia prevalence: a systematic review. Oral Oncol. 2003; 39(8): 770-80.

40. Lee JJ, Hung HC, Cheng SJ, Chen YJ, Chiang CP, Liu BY, Jeng JH, Chang HH, Kuo YS, Lan WH, Kok SH. Carcinoma and dysplasia in oral leukoplakias in Taiwan: prevalence and risk factors. Oral Surg Oral Med Oral Pathol Oral Radiol Endod. 2006 Apr; 101(4): 472-80.

41. Van der Waal I. Potentially malignant disorders of the oral and oropharyngeal mucosa. Present concepts of management. Oral Oncol. 2010; 46(6): 423-5.

42. Neville BW, Day TA. Oral cancer and precancerous lesions. CA Cancer J Clin. 2002; 52(4): 195-215.

43. Boy SC. Leukoplakia and erythroplakia of the oral mucosa--A brief overview. SADJ. 2012; 67: 558-60.

44. Moret Y, Rivera H, González JM. Correlación clínico-patológica de lesiones diagnosticadas inicialmente como leucoplasia bucal y el diagnóstico histopatológico de displasia epitelial en una muestra de 11.250 pacientes adultos. Facultad de Odontología. U.C.V. Acta Odontol Venez. 2008; 46(3): 265-8.

45. Van der Waal I, Axéll T. Oral leukoplakia: a proposal for uniform reporting. Oral Oncol. 2002; 38(6): 521-6.

46. Villa A, Villa C, Abati S. Oral cancer and oral erythroplakia: an update and implication for clinicians. Aust Dent J. 2011; 56(3): 253-6.

47. Yardimci G, Kutlubay Z, Engin B, Tuzun Y. Precancerous lesions of oral mucosa. World J Clin Cases. 2014 Dec 16; 2(12): 866-72. 
48. Estrada Pereira GA, Zayas Simón OP, González Heredia E, González Alonso C, Castellanos Sierra G. Diagnóstico clínico e histopatológico de la eritroplasia bucal. Medisan [internet]. 2010 Jun; 14(4): 433-8. Disponible en: h ttps://www.redalyc.org/articulo.oa?id=368445240003

49. Reichart PA, Phillipsen HP. Oral erythroplakia--a review. Oral Oncol. 2005; 41(6): 551-61.

50. Noonan VL, Kabani S. Diagnosis and management of suspicious lesions of the oral cavity. Otolaryngol Clin North Am. 2005; 38(1): 21-35.

51. Hernández Osorio C, Fuentes Palma B, Cartes-Velásquez R. Queilitis actínica: aspectos histológicos, clínicos y epidemiológicos. Rev Cub Estomatol. 2016; 53(2): 45-55.

52. de Santana Sarmento DJ, da Costa Miguel MC, Queiroz LM, Godoy GP, da Silveira EJ. Actinic cheilitis: clinicopathologic profile and association with degree of dysplasia. Int J Dermatol. 2014 Apr; 53(4): 466-72.

53. Orozco P, Vásquez $S$, Venegas B, Rivera C. Prevalencia de queilitis actínica en trabajadores expuestos a radiación ultravioleta en Talca, Chile. Rev Clin Periodoncia Implantol Rehabil Oral. 2013; 6(3): 127-9.

54. Sánchez Ferra D, Alcalá Pérez D, Peralta Pedrero ML, Vega González M, Medina Bojórquez A, Valenzuela Flores $\mathrm{AB}$, Torres Arreola LP. Guía de práctica clínica para diagnóstico y tratamiento de la queratosis actínica. Dermatol Rev Mex. 2012; 56(1): 14-25.

55. de Santana S, da Costa M, Queiroz L, Godoy G, da Silveira E. Actinic cheilitis: clinicopathologic profile and association with degree of dysplasia. Int J Dermatol. 2014; 53(4): 466-72.

56. Ardila C. Revisión sistemática de los efectos del hábito de fumar invertido sobre la mucosa oral. Arch Med Camagüey. 2013; 17(3): 405-15.

57. Vargas-Ferreira F, Nedel F, Etges A, Gomes AP, Furuse C, Tarquinio SB. Etiologic factors associated with oral squamous cell carcinoma in non-smokers and non-alcoholic drinkers: a brief approach. Braz Dent J. 2012; 23(5): 586-90.

58. Gamboa M, Villarroel-Dorrego M. Características clínicas e histopatológicas y expresión de p53 en lesiones palatinas producidas por tabaquismo de forma invertida. Cienc Odontol. 2001; 10(1):36-42.

59. Gutiérrez P, Olivares R, Leyva ER. Factor de crecimiento epidermal y proteínas totales en saliva de fumadores y no fumadores. Av Odontoestomatol. 2008; 24(6): 377-83.

60. Álvarez-Gómez GJ, Álvarez-Martínez E, Jiménez-Gómez R, Mosquera-Silva Y, Gaviria-Núñez AM, GarcésAgudelo A, Alonso-Duque A, Zabala-Castaño A, Echeverri-González E, Isaac-Millán M, Ramírez-Ossa D. Reverse smokers's and changes in oral mucosa. Department of Sucre, Colombia. Med Oral Patol Oral Cir Bucal [internet]. 2008 Jan 1; 13(1): E1-8. Disponible en: http://www.medicinaoral.com/pubmed/medoralv13_i1_p 1.pdf

61. Pérez MA, Raimondi AR, Itoiz ME. An experimental model to demonstrate the carcinogenic action of oral chronic traumatic ulcer. J Oral Pathol Med. 2005 Jan; 34(1): 17-22.

62. Piemonte ED, Lazos JP, Brunotto M. Relationship between chronic trauma of the oral mucosa, oral potentially malignant disorders and oral cancer. J Oral Pathol Med. 2010; 39(7): 513-7.

63. Balkwill, FR, Mantovani A. Cancer-related inflammation: common themes and therapeutic opportunities. Sem Cancer Biol. 2012; 22(1): 33-40.

64. Somacarrera Pérez ML, López Sánchez AF, Martín Carreras-Presas C, Díaz Rodríguez M. Lesiones traumáticas en la mucosa oral de los adultos mayores. Av Odontoestomatol. 2015; 31(3): 129-34.

65. Miranda R, Cuba S, Gamarra A. Úlcera en lengua causada por remanentes radiculares: Reporte de caso. Rev Med Hered. 2014; 25(4): 223-6.

66. Lazos JP, Piemonte ED, Lanfranchi HE, Brunotto MN. Characterization of chronic mechanical irritation in oral cancer. Int J Dent. 2017: 6784526.

67. Valente VB, Takamiya AS, Ferreira LL, Felipini RC, Biasoli ÉR, Miyahara GI, Bernabé DG. Oral squamous cell carcinoma misdiagnosed as a denture-related traumatic ulcer: A clinical report. J Prosthet Dent. 2016 Mar; $115(3): 259-62$. 


\section{Notas}

* Artículo de revisión.

Licencia Creative Commons CC BY 4.0

Cómo citar este artículo: Tovío Martínez EG, Carmona Lorduy MC, Díaz-Caballero AJ, Harris Ricardo J, Lanfranchi Tizeira HE. Expresiones clínicas de los trastornos potencialmente malignos en la cavidad oral. Revisión integrativa de la literatura. Univ Odontol. 2018 ene-jun; 37(78). https://doi.org/10.11144/Javer iana.uo37-78.ecdp 